\title{
ENTORNO FAMILIAR DURANTE LA NIÑEZ Y LA COMISIÓN DE DELITO EN POBLACIÓN PENITENCIARIA PERUANA
}

\author{
FAMILY ENVIRONMENT DURING CHILDHOOD AND THE \\ COMMISSION OF CRIME IN THE PERUVIAN \\ PRISON POPULATION
}

\author{
Julio Cjuno', Giulianna Gamero-Vega', \\ María Teresa Reyes Alvarez $z^{3}$ Víctor Dulanto ${ }^{4}$
}

\section{RESUMEN}

El presente estudio tuvo el objetivo de determinar el análisis de correspondencia entre del entorno familiar en la niñez y la comisión de delito en población penitenciaria peruana. Se trata de un estudio de análisis de correspondencias múltiples, multivariado para analizar la relación y la agrupación que existe entre género, entorno familiar en la niñez y comisión de delito, utilizando la base de datos del primer Censo Nacional de Población Penitenciario 2016 realizado por el Instituto Nacional de Estadística e Informática INEI. De los 76 mil 180 prisioneros de población penitenciaria a nivel nacional, se seleccionó a los presos con sentencia por haber cometido un delito y que fueran peruanos por nacimiento. Se obtuvo la cantidad de 9 mil 621 presos que cumplieron con los criterios de selección, la edad media fue de 35,21, el $95,8 \%$ son hombres y el 4,2\% mujeres, el 47,5\% reportó que es soltero, el 36,8\% conviviente. Encontramos que existe una correspondencia entre los eventos adversos "sus padres le pagaban" y "consumían alcohol”, mientras que el delito de "estafa" se agrupa con "mujeres prisio-

1 Instituto de Investigación IIU, Universidad Católica Los Ángeles de Chimbote, Ancash, Perú.

2 Instituto de Investigación IIU, Universidad Católica Los Ángeles de Chimbote, Ancash, Perú.

3 Jefe del Centro de Investigación SANNA, Clínica Sánchez Ferrer, Trujillo, Perú.

4 Instituto Nacional de Oftalmología, Lima, Perú. 
neras" representa un nuevo hallazgo; no hay estudios que reporten aquello. Por último se agrupó también el evento adverso "en la niñez sus padres le pegaban" con el delito de "robo, hurto en grados desde robo simple hasta agravado". Concluyeron que violencia en la niñez genera problemas mentales y de personalidad, graves en el adulto, conduciendo a conductas antisociales, es necesario realizar campañas preventivas y educativas a padres violentos con sus hijos.

PALABRAS CLAVE: Comisión del delito, INEI, población penitenciaría, presos.

\section{ABSTRACT}

In this study, the objective was to determine the analysis of correspondence between the family environment in childhood and the commission of crime in the Peruvian penitentiary population. It is a multivariate analysis of multiple correspondences analysis to analyze the relationship and the grouping that exists between gender, family environment in childhood and commission of crime, using the database of the first National Penitentiary Population Census 2016 conducted by the National Institute of Statistics and Informatics INEI. Of the 76 thousand 180 prisoners of prison population nationwide, prisoners were selected with a sentence for having committed a crime and were Peruvian by birth. The number of 9,621 prisoners who met the selection criteria was obtained, the average age was 35.21, 95.8\% were men and $4.2 \%$ were women, $47.5 \%$ reported being single, $36.8 \%$ were cohabiting. We found that there is a correspondence between the adverse events "his parents paid him" and "they consumed alcohol", while the crime of "swindling" is grouped with "female prisoners" represents a new finding; there are no studies that report that. Finally, the adverse event was also grouped "in childhood his parents beat him" with the crime of "robbery, robbery in grades from simple robbery to aggravated". They concluded that violence in childhood generates mental and personality problems, serious in the adult, leading to antisocial behavior, it is necessary to carry out preventive and educational campaigns for violent parents with their children.

KEY WORDS: Commission of crime, INEI, prison population, prisoners.

\section{INTRODUCCIÓN}

Según el último reporte mundial de población penitenciaria de la International Centre for Prisons Studies (ICPS) existe un reporte de prevalencia mayor a 10 millones de prisioneros en todo el mundo; de esta población, casi la mitad de presos se encuentra distribuida entre Estados Unidos, Rusia y China. El crecimiento de esta población mundial en los últimos 15 años fue de 25 $30 \%$; sin embargo, en países americanos el crecimiento fue del $83 \%$. Sin lugar a duda, este crecimiento es casi tres veces mayor en comparación a países de otros continentes. 
En países latinoamericanos como Brasil hay más de 580 mil personas privadas de libertad en las cárceles, siendo acreedores de la mayor población de presos en esta región ${ }^{2}$. En Colombia hasta el 2007 existían 61 mil 133 presos, en condiciones de hacinamiento, pobreza y discriminación ${ }^{3}$. En Chile existen más de 46 mil 825 presos en todos los centros penitenciarios ${ }^{4}$. En Perú según el último reporte del Instituto Nacional de Estadística e Informática (INEI) existen 76 mil 180 prisioneros ${ }^{5}$.

Las razones por las cuales fueron privados de libertad varían en cada región y país según las leyes, debido a que las leyes varían por diferentes factores políticos, cultura y principios. En el código procesal penal peruano el delito está clasificado en diversos tipos de delitos según su gravedad y clasificaciones adicionales descritas en decreto legislativo peruano ${ }^{6}$.

Encontrar el origen de la comisión del delito ha sido la interrogante de diversos investigadores. Tal es el caso de un estudio con 540 presos varones del distrito de Abruzzo-Molise, Italia, encontraron que el trauma infantil es un factor determinante de la agresividad de los presos ${ }^{7}$. Otro estudio, en una muestra masculina de prisioneros fillandeses reportó que el abuso de manera física y sexual era más común en mujeres que en hombres, sin embargo, la integridad familiar y el consumo de drogas de sus padres influían más en presos varones .

A diferencia de presos varones, en mujeres presas del sistema penitenciario de Texas, Estados Unidos, se ha encontrado que el 40\% de las mujeres fueron diagnosticadas como dependientes al alcohol y tenían mayores probabilidades de haber crecido en familias disfuncionales, con consumo de drogas y alcohol por sus padres, además de haber experimentado el abuso físico y sexual en su infancia?. Otro estudio con mujeres encarceladas en Noruega encontraron que el abuso emocional, físico y sexual experimentado en la niñez, más el abandono emocional y físico por sus padres incrementó significativamente el riesgo de intento suicida y abuso de drogas ${ }^{10}$.

Las experiencias de los prisioneros vividas en la niñez en relación con la violencia, drogas, alcohol y otros factores, ha influido en sus decisiones para llegar a cometer algún tipo de delito, debido a que además, han desarrollado una amplia gama de problemas psicopatológicos que no les ha permitido actuar de manera coherente ante la infracción de la ley, y debido a ello, hoy se encuentran privados de libertad ${ }^{11}$.

Un estudio realizado en una prisión de Reino Unido se relacionó los múltiples eventos adversos en la niñez, con el primer delito cometido en mujeres, encontraron que estas se asociaban de forma significativa $(p=0,001)^{12}$. 
$\mathrm{Al}$ respecto, no se ha realizado ningún estudio sobre el entorno familiar en la niñez y la comisión del delito en presos peruanos; es por ello que este estudio tuvo el objetivo de determinar el análisis de correspondencia que existe entre del entorno familiar en la niñez y la comisión de delito en población penitenciaria peruana.

\section{MATERIALES Y MÉTODOS}

Se utilizó la base de datos del primer Censo Nacional de Población Penitenciario 2016 realizado por el Instituto Nacional de Estadística e Informática INEI ${ }^{5}$, cuyo censo tuvo como objetivo generar información cuantitativa y cualitativa actualizada sobre las características socioeconómicas de la población penitenciaria, con la intención de implementar y ejecutar diversos programas de apoyo para una adecuada reinserción de los presos en el entorno laboral y social.

\section{POBLACIÓN Y MUESTRA}

\section{Población}

De los 76 mil 180 prisioneros que representa el reporte total de población penitenciaria a nivel nacional, se realizó una selección de los presos que se encuentran sentenciados por haber cometido un delito y que fueran peruanos por nacimiento. Se obtuvo la cantidad de 9621 presos que cumplieron con los criterios de selección. Para ello se realizó una representación de esquema de muestra seleccionada.

\section{Tamaño de muestra}

Para el cálculo de muestra utilizamos el programa estadístico Epidat 3.1 con una población total de 9621 para una proporción esperada de 55\% del principal delito con mayor frecuencia y un nivel de confianza de $95 \%$ con una precisión absoluta mínima de $1 \%$ máxima de $5 \%$ con incrementos de $1 \%$ y con efecto diseño de 1 . Utilizamos la precisión del $3 \%$ para obtener el resultado de tamaño de muestra representativa de 952 presos.

\section{Variables de estudio}

Se reporta como variables sociodemográficas: Edad, estado civil y género. La edad se midió en función al número de años en edad cronológica de los presos. El estado civil está dado en seis categorías (conviviente, casado, viudo, divorciado, separado, soltero). El sexo se clasificó en hombre y mujer. 


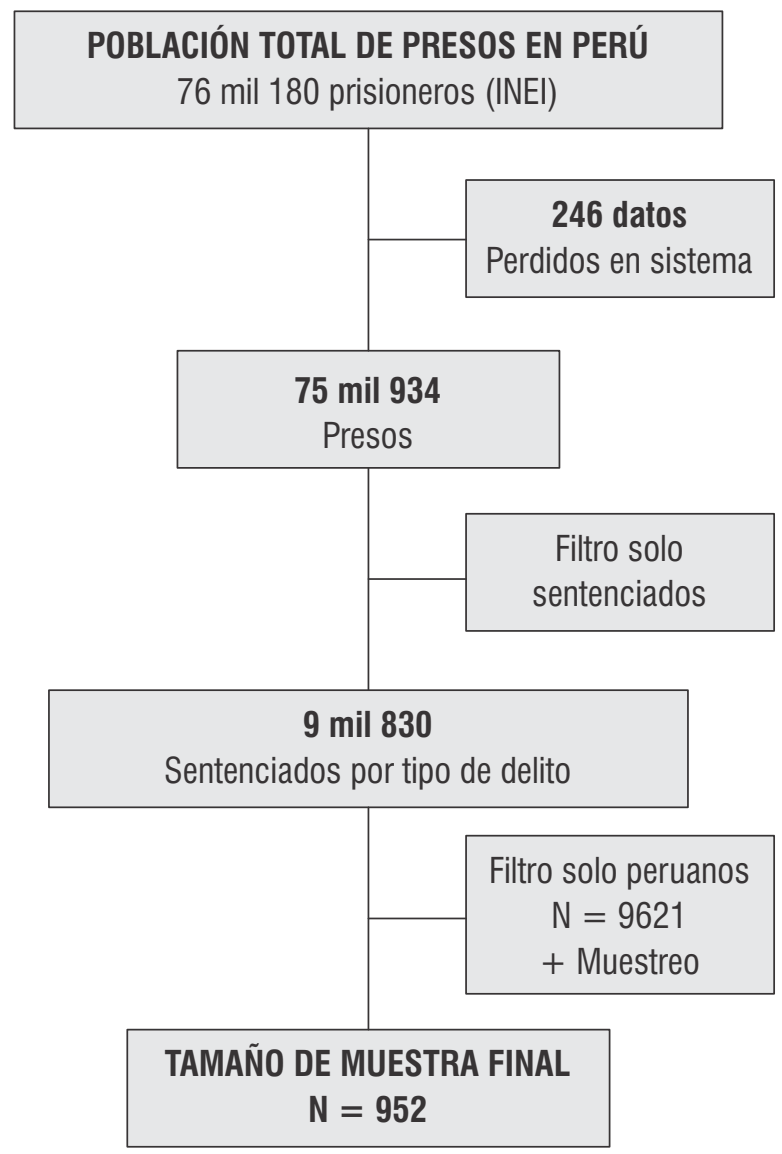

Figura 1. Esquema de selección de población peruana de presos.

Se realizó la selección de población nacida en Perú, mediante la pregunta país de nacimiento, corroborado con nacionalidad peruana. Se realizó la selección de preguntas para sentenciados. Se consideró como tipo de delito al motivo de sentencia dividido en: homicidio doloso / asesinato, homicidio culposo, secuestro, lesiones, delitos sexuales, robo/robo agravado, delitos contra la salud, usurpación, tenencia ilegal de arma, hurto/hurto agravado, estafa/ apropiación ilícita, extorsión.

Se seleccionaron las preguntas que cuestionan entorno familiar en la niñez (5-12 años), las cuales cuestionaban los siguientes temas: ¿Cuándo usted era niño(a) sus padres o personas que asumieron ese rol le pegaban?, ¿Cuándo usted 
era niño(a), sus padres o adultos que vivían con usted, consumían droga?, ¿Cuándo usted era niño(a) algunos de sus padres o adultos que vivían con usted consumían droga?

\section{Análisis de datos}

Estudio de análisis de correspondencias múltiples, multivariado para analizar la relación y la agrupación que existe entre género, entorno familiar en la niñez y comisión de delito. Se utilizó el programa SPSS Statistics IBM versión 22.0

\section{RESULTADOS}

Tabla 1

CARACTERÍSTICAS DEMOGRÁFICAS DE LA MUESTRA DE POBLACIÓN PENITENCIARIA PERUANA.

\begin{tabular}{lcr}
\hline & Población penitenciaria $(\mathrm{N}=924)$ & $\%$ \\
\hline Edad & $35.21(11.31)$ & \\
Media (DE) & & \\
Sexo & 885 & 95,8 \\
Hombre & 39 & 4,2 \\
Mujer & & \\
Estado civil & 340 & 36,8 \\
Conviviente & 99 & 10,7 \\
Casado(a) & 11 & 1,2 \\
Viudo(a) & 9 & 1,0 \\
Divorciado(a) & 26 & 2,8 \\
Separado(a) & 439 & 47,5 \\
Soltero(a) & & \\
\hline
\end{tabular}

Fuente: Base de datos del Censo Nacional de Población Penitenciaria 2016- INEI

Contamos con una muestra poblacional de 924 presos de ellos, con una edad media de 35,21, el 95,8\% son hombres y el 4,2\% mujeres, el 47,5\% reportó que es soltero, el $36,8 \%$ conviviente (tabla 1 ). 


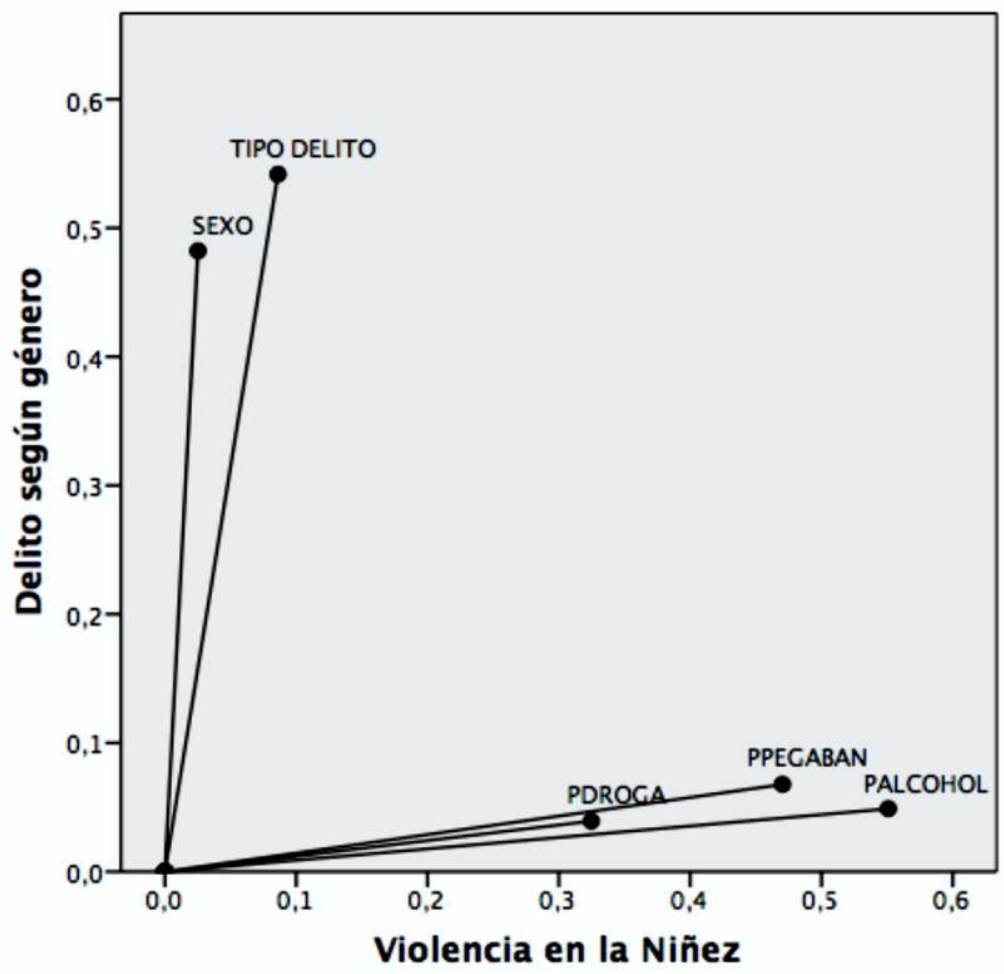

Leyenda: SEXO: masculino o femenino.

TIPO DE DELITO: descrito en variables.

PDROGA: Padres consumían droga.

PPEGABAN: sus padres le pegaban en la infancia.

PALCOHOL: sus padres consumían alcohol.

Fuente: Base de datos del Censo Nacional de Población Penitenciaria 2016- INEl.

Figura 2. Correspondencias entre tipo de delito según género y violencia en la niñez por dimensiones.

Encontramos que las dimensiones se agrupan entre el género y el tipo de delito en los presos de población penitenciaria peruana; asimismo, el evento adverso en la niñez de que sus padres o apoderados le pegaban y sus padres consumían alcohol se agrupan, mientras que el evento adverso en la niñez de que sus padres consumían drogas se mantiene alejado de ambos grupos anteriormente mencionados. 


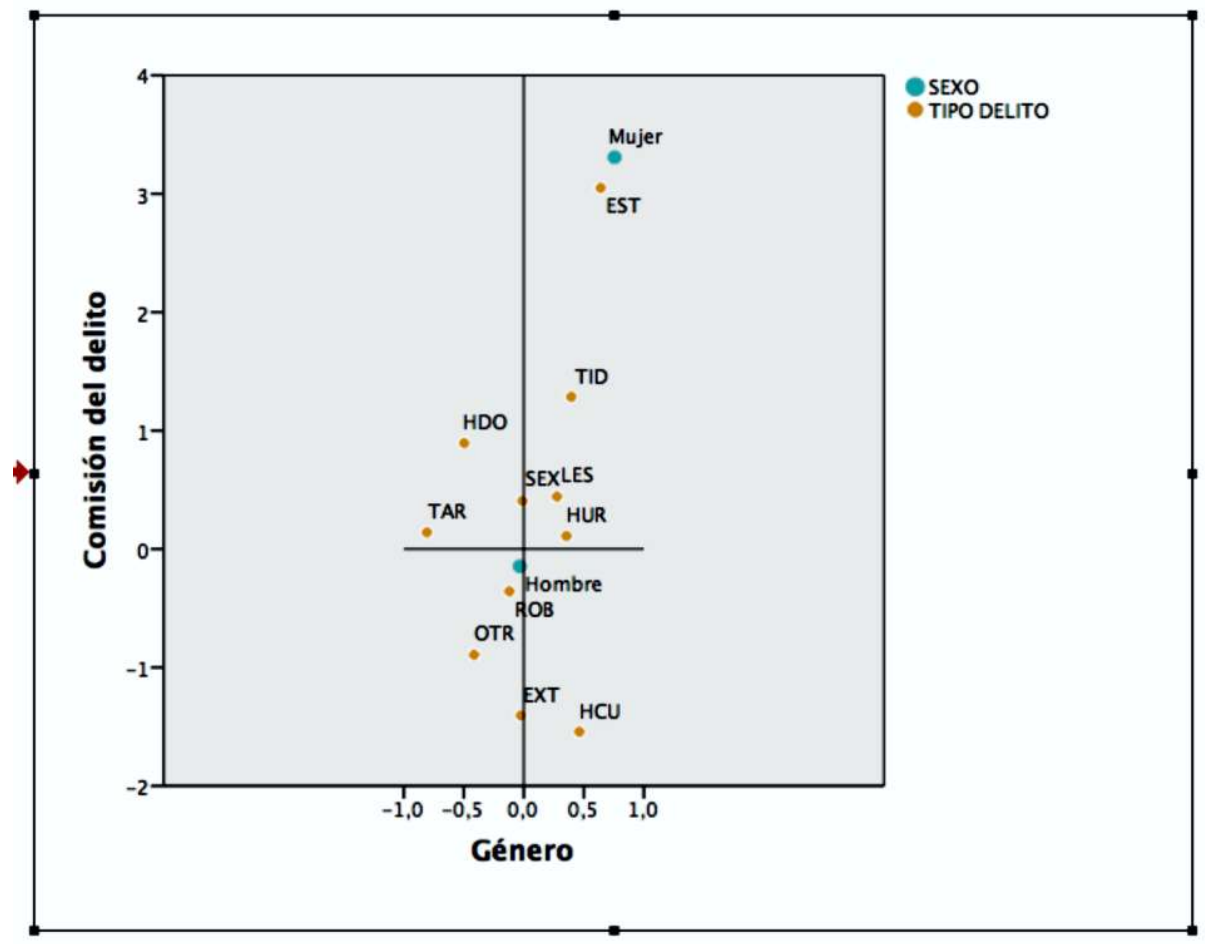

Leyenda: Tipo de delito: Homicidio Doloso = HDO.

Homicidio Culposo $=\mathrm{HCU}$.

Secuestro $=$ SEC .

Lesiones $=$ LES .

Delitos sexuales $=$ SEX.

Robo $/$ Robo agravado =ROB.

Tráfico llícito de drogas $=$ TID.

Usurpación = USU.

Tendencia ilegal de armas $=$ TAR.

Hurto/hurto agravado $=$ HUR .

Estafa/ Apropiación ilícita = EST .

Otros = OTR.

Extorsión $=$ EXT.

Fuente: Base de datos del Censo Nacional de Población Penitenciaria 2016- INEI.

Figura 3. Gráfico por correspondencias múltiples del tipo de delito según sexo (hombre/mujer)

Se aprecia la agrupación de la percepción del género, donde se observa una mayor relación de las mujeres con el delito de estafar, y de los hombres con el delito por robo, hurto y de otros tipos de delitos. 


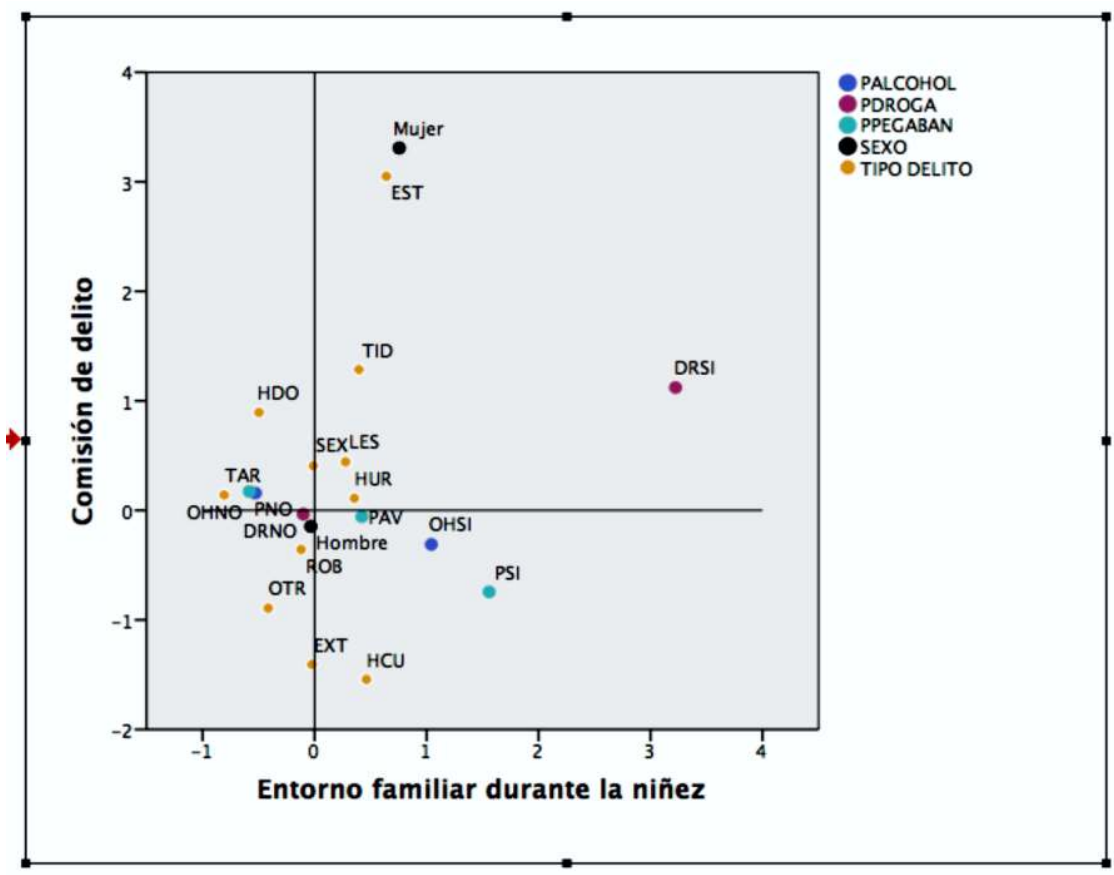

Leyenda: Homicidio Doloso $=$ HDO.

Homicidio Culposo $=\mathrm{HCU}$.

Secuestro $=$ SEC

Lesiones $=$ LES.

Delitos sexuales $=$ SEX.

Robo $/$ Robo agravado $=$ ROB

Tráfico llícito de drogas $=$ TID .

Usurpación = USU .

Tendencia ilegal de armas $=$ TAR

Hurto/hurto agravado $=$ HUR .

Estafa/ Apropiación ilícita $=$ EST

Otros $=$ OTR.

Extorsión $=$ EXT
Padres le pegaban siempre $=$ PSI .

Padres le pegaban a veces $=$ PAV.

Padres no le pegaban $=$ PNO.

Padres consumían alcohol $=\mathrm{OHSI}$

Padres no consumían alcohol $=$ OHNO.

Padres consumían drogas $=$ DRSI .

Padres no consumían drogas = DRNO.

Fuente: Base de datos del Censo Nacional de Población Penitenciaria 2016- INEI.

Figura 4. Gráfico de correspondencias múltiples con el tipo de delito cometido, según sexo (hombre/mujer) y tipo de entorno agresivo vivido en la infancia (padres que les pegaban, consumían drogas o bebidas alcohólicas).

Se observa que persiste la relación de las mujeres con el delito de estafar, mientras que en los hombres se agrupan más entre sus padres le pegaban y la relación con la comisión de robar, hurtar y otros tipos de delito; mientras que la variable de sus padres consumían drogas se agrupa con robo. 


\section{ANÁLISIS DE RESULTADOS}

Contamos con una muestra poblacional de 924 presos de ellos, con una edad media de 35,21 , el 95,8\% son hombres y el 4,2\% mujeres, el 47,5\% reportó que es soltero, el $36.8 \%$ conviviente (tabla 1). Evidentemente existe una diferencia poblacional por sexo, sin embargo, un estudio realizado en Bucaramanga, Colombia encontró que el $14,54 \%$ de los presos eran del sexo femenino ${ }^{13}$, dato similar o cercano a la realidad que describimos en nuestro estudio. Esta razón se asume que este reporte es frecuente en estudios con presos.

En referencia a las correspondencias analizadas en este estudio, encontramos que el evento adverso en la niñez de que sus padres le pegaban y sus padres consumían alcohol se agrupan (figura 2). Pues un estudio realizado en Argentina refuerza este hallazgo, pues indica que el $91 \%$ de los presos de una cárcel reportaron alguna experiencia adversa en la niñez, el 59\% fue agresión física, 54\% daño emocional, $18 \%$ abuso sexual, abandono o negligencia $66 \%$, además se supo que la mayoría de estos abusos lo recibieron de algún miembro de su hogar ${ }^{14}$. Si volvemos a nuestro resultado, entonces podríamos decir que consumir alcohol podría conducir a pegar a sus hijos a padres de familia en general, por lo tanto, esto estaría generando el inicio de una vida delictiva en sus hijos. Mientras que el evento adverso en la niñez de que sus padres consumían drogas se mantiene alejado de ambos grupos anteriormente mencionados. Esto podría ser debido a que estas personas necesitan aislarse para poder consumir sus drogas.

A diferencia de la descripción anterior, en la figura 3 visualizamos la agrupación de la percepción del género, donde se observa una mayor relación de las mujeres con el delito de estafar, y de los hombres con el delito por robo, hurto y de otros tipos de delitos. Hacemos referencia a la mujer prisionera peruana, tal vez el motivo por el que no hemos podido encontrar estudios que aporten a que las mujeres generalmente cometen el delito de estafa en otros países se deba características propias de las prisioneras peruanas, tales como delitos cometidos por necesidad, debido a la pobreza de donde provienen estas mujeres en su mayoría.

Sobre la relación de las mujeres con el delito de estafar (figura 4) encontrado en nuestro estudio es reforzado por un estudio realizado en Zirich, Suisa, que los principales motivos por los cuales las mujeres fueron condenadas fue por asesinatos, robos u solo uno por delito sexual; además que las mujeres prisioneras tienen baja educación, sufrieron experiencias adversas en la niñez y no tenían buena salud mental ${ }^{15}$. En nuestro estudio, algunas características tales como el bajo 
nivel de educación, vivir en una situación de desventaja y lleno de eventos traumáticos durante su infancia podrían haberlas iniciado en el delito de la estafa en el caso de la realidad de las mujeres encarceladas peruanas.

Mientras que en los hombres se agrupan más entre sus padres le pegaban y la relación con la comisión de robar, hurtar y otros tipos de delito. La asociación entre el evento adverso en la niñez de que sus padres le pegaban, ya se había dado de una manera similar en un estudio donde los investigadores llegaron a la conclusión de que la trauma infantil es un determinante en la agresividad del prisionero?.

\section{LIMITACIONES DEL ESTUDIO}

Son limitaciones propias de estudios realizados con base de datos secundarios, como es el caso de nuestra limitación en el análisis es en función a datos que fueron recolectados con otro fin (estudio primario); debido a ello la información disponible no brinda información como desearíamos de una especificación en todos los presos y el primer delito cometido.

La pregunta dirigida a consumo de alcohol en los padres, es poco específica, ya que puede ser interpretada como un consumo esporádico o frecuente, pero sin llegar a la embriaguez o al consumo que genere dependencia, de tal manera que ocasione la disrupción en la vida laboral, familiar y social.

\section{CONCLUSIONES}

Existe una correspondencia entre los eventos adversos de sus padres le pagaban y consumían alcohol, mientras que el delito de estafa se agrupa con mujeres prisioneras representa un nuevo hallazgo; no hay estudios que reporten aquello. Por último se agrupó también el evento adverso en la niñez de sus padres le pegaban con el delito de robo, hurto en grados desde robo simple hasta agravado.

Ante el hallazgo de que la violencia en la niñez genera problemas mentales y de personalidad graves en el adulto, conduciendo a conductas antisociales, es necesario realizar campañas preventivas y educativas a padres violentos con sus hijos.

Las autoridades peruanas y tomadores de decisiones deben generar políticas educativas orientadas a disminuir la violencia en la niñez percibida de parte de sus progenitores. 


\section{REFERENCIAS BIBLIOGRÁFICAS}

1. Walmsley R. World Prison Population List (eighth edition). London WC2B; 2008.

2. Bourdillon PM, Gonçalves CCM, Pelissari DM, Arakaki-Sanchez D, Ko AI, Croda J, et al. Increase in Tuberculosis Cases among Prisoners, Brazil, 2009-2014(1). Emerg Infect Dis. marzo de 2017;23(3):496-9.

3. Gomez IT, Llerena CR, Zabaleta AP. Tuberculosis y tuberculosis farmacorresistente en personas privadas de la libertad. Colombia, 2010-2012. Rev Salud Pública. octubre de 2015;17(1):97-105.

4. Mundt AP, Alvarado R, Fritsch R, Poblete C, Villagra C, Kastner S, et al. Prevalence rates of mental disorders in Chilean prisons. PLoS One. 2013;8(7):e69109.

5. Instituto Nacional de Estadística e Informática, Ministerio de Justicia y Derechos Humanos, Instituto Nacional Penitenciario. Perú: Primer Censo Nacional Penitenciario - Perfíl de la población del penal. 2016.

6. Contraloria General de la República-Perú. Código penal Decreto lesgislativo Nº 635 [Internet]. Lima; 2008. Disponible en:

https://www.unifr.ch/ddp1/derechopenal/legislacion/__20080616_75.pdf

7. Sarchiapone M, Carli V, Cuomo C, Marchetti M, Roy A. Association between childhood trauma and aggression in male prisoners. Psychiatry Res. enero de 2009;165 (1-2):187-92.

8. Viitanen P, Vartiainen H, Aarnio J, von Gruenewaldt V, Lintonen T, Mattila AK, et al. Childhood maltreatment and mental disorders among Finnish prisoners. Int J Prison Health. agosto de 2011;7(4):17-25.

9. Mullings JL, Hartley DJ, Marquart JW. Exploring the relationship between alcohol use, childhood maltreatment, and treatment needs among female prisoners. Subst Use Misuse. enero de 2004;39(2):277-305.

10. Friestad C, Åse-Bente R, Kjelsberg E. Adverse childhood experiences among women prisoners: Relationships to suicide attempts and drug abuse. Int J Soc Psychiatry. febrero de 2014;60(1):40-6.

11. Villagra P, Fernandez P, Garcia-Vega E, Gonzalez-Menendez A. Dual Diagnosis in Prisoners: Childhood Sexual and Physical Abuse as Predictors in Men and Women. Int J Offender Ther Comp Criminol. noviembre de 2013;

12. Karatzias T, Power K, Woolston C, Apurva P, Begley A, Quinn C, et al. Multiple traumatic experiences, post-traumatic stress disorder and offending behaviour in female prisoners. Crim Behav Ment Heal. 2017;

13. Larrotta Castillo R, Gómez Camacho M, Beltrán Gómez CA. Modus operandi en personas recluidas por el delito de hurto en prisiones de Bucaramanga, Colombia. Inf psicológicos [Internet]. 1 de septiembre de 2017 [citado 10 de noviembre de 2017];17(2):107-18. Disponible en:

https://revistas.upb.edu.co/index.php/informespsicologicos/article/view/7625 
14. Vallejo M, Bertone MS. [Traumatic childhood in a private male population of liberty events: prevalence study]. Rev Fac Cien Med Univ Nac Cordoba [Internet]. 2016 [citado 10 de noviembre de 2017];73(2):75-82.

Disponible en: http://www.ncbi.nlm.nih.gov/pubmed/27420141

15. Rossegger A, Wetli N, Urbaniok F, Elbert T, Cortoni F, Endrass J. Women convicted for violent offenses: Adverse childhood experiences, low level of education and poor mental health. BMC Psychiatry [Internet]. 22 de diciembre de 2009 [citado 10 de noviembre de 2017];9(1):81. Disponible en: http://www.ncbi.nlm.nih.gov/pubmed/20028499 